\title{
Emergency Department Nurses' Perceptions toward Factors Influencing the Occurrence of Medication Administration Errors
}

\section{Naglaa Abd El- Aziz El Seesy* and Faten El Sebaey}

Alexandria University, Department of Nursing Administration, Faculty of Nursing , Alexandria , Egypt

*Corresponding author: Naglaa Abd El- Aziz El Seesy, Alexandria University, Department of Nursing Administration, Faculty of Nursing , Alexandria ,Egypt, Tel: +201222535059; E-mail: nona20102002@ yahoo.com

Received date: May 15, 2015; Accepted date: Aug 11, 2015; Published date: Aug 19, 2015

Copyright: ( 2015 El Seesy et al. This is an open-access article distributed under the terms of the Creative Commons Attribution License, which permits unrestricted use, distribution, and reproduction in any medium, provided the original author and source are credited.

\begin{abstract}
Background: Significant efforts have been directed to understand medication errors causes in recent years because it contributes directly to patient morbidity and mortality. This study was conducted to determine the factors influencing the occurrence of medication administration errors, as perceived by nurses in emergency department (ED).
\end{abstract}

Aim: The current study aimed to assess emergency department nurses' perceptions toward factors influencing the occurrence of medication administration errors.

Design: The study followed a cross-sectional descriptive design.

Setting: The present study was carried out at ED in teaching Main University Hospital in Alexandria governorate, Egypt.

Subjects: 84 nursing staff worked in the previous mentioned setting.

Tool: The data gathering tool was Medication Administration Errors (MAEs) Reporting Questionnaire which was developed by Wakefield in 1998 [1]. It contains 16 items regarding reasons why medication errors occur.

Results: This study suggested four categories for reasons of why MAEs occur in emergency department and the leading cause of medication errors was due to nurses- physicians' communication.

Conclusion: Medication errors are common in emergency department. A wide range of factors perceived as contributing factors of the occurrence of medication administration errors were identified such as nurses- physicians communication, medication packaging, pharmacy processes and nurse staffing. This information could be used to improve the medication system in emergency department in Egypt.

Recommendations: This study recommended for provision of safe work environment that encourage good physicians-nurses team work relationship, dissemination of safety guidelines in all hospital department specially nursing and pharmacy, on-going education and training on safe medication administration and supervision of newly hired nursing staff during medication administration process and provision of adequate staffing and fair scheduling for nursing staff working in a highly urgent care departments.

Keywords: Medication administration errors; Emergency department; Nurses; Perception

\section{Introduction}

MAE is one of the factors causing death and harm to patients and the most common important challenges threatening healthcare system in all countries worldwide [2]. The definition typically cited in literature that is authored by nurses defines MAE as mistakes associated with drugs and intravenous solutions that are made during the prescription, transcription, dispensing, and administration phases of drug preparation and distribution [1,3]. Medication administration errors (MAEs) in the ED are common, with errors occurring most often in the prescribing and administration phase [4].
American Society of Health System Pharmacists (ASHP), (2003) recognizes that medication errors can be minimized by assessing the medication use process, identifying inadequacies within systems, and developing interventions to correct the recognized deficiencies.

A possible contributing factor to MAEs in the ED is the unique medication distribution system used. For example, on-pharmacy profiled automated dispensing cabinet (ADC), unit stock, or refrigerator, as the prescriber's order may not be reviewed by a pharmacist before the drugs are given (Flynn et al. 2010). However, high patient acuity, crowding, and frequent interruptions are pervasive in the ED's clinical environment. Interruptions in the ED are conservatively estimated to be as frequent as every minute for attending physicians and every 14 minutes for resident physicians. 
Although the results are mixed, several studies suggest that links exist between medication error and systemic organizational factors. These include nurse staffing adequacy, hours worked per week, overtime, staffing mix (professional versus unregulated), and other factors reflecting how the work system is designed [5-7]. Evidence of links between stress in the clinical workplace and medication error is also emerging. For example, a recent study of nurses in Alberta and Ontario found that patient safety outcomes including medication error and other adverse events were associated with emotional exhaustion ('burnout') in nurses, which in turn was related to staffing inadequacy, poor nurse-physician relations, and other "work life" factors [8]. Clearly, adverse drug events that occur in the ED are a significant public health problem and need to be reduced, but this must be accomplished without making the ED less efficient [9].

Previous studies have examined important factors in refusal or act as barriers to report medication errors [10-13] or the analysis and improvement medication error reporting practices by emergency department physicians, nurses, and pharmacists [14]. However, few studies have focused only on the assessment of the occurrence and reasons of nursing medication errors, these studies recommended further studies to determine and investigate the causes of medication errors [15-18].

Understanding factors that contribute to medication error is the first step toward preventing it to ensure safety and quality of patient care. Therefore we have conducted this study to explore the most important factors influencing the occurrence of MAEs in ED from nurses' perspective which can lead to improve medication administration process, diminish the risks of adverse events that impact patient morbidity and mortality, improve patient safety, and lower cost of patient care.

\section{Materials and Methods}

\section{Research Design}

This study followed a cross-sectional descriptive design.

\section{Setting}

This study was carried out in emergency department at teaching Main University Hospital in Alexandria governorate, Egypt which contains 42-bed during the period from June 2013 to the end of august 2013.

\section{Subjects}

All nursing staff $(n=84)$ who were working in the previously mentioned setting and willing to participate in such study were included. All of them were responsible for providing nursing care for urgent cases.

\section{Tool}

Medication Administration Error Survey: It was developed by Wakefield in 1998 [1] included 16 items regarding reasons why medication errors occur. Respondents were asked to how much they believe they affect the occurrence of medication errors in the emergency department using a six points Likert type scale with fix values ranging from $6=$ strongly agree to $1=$ strongly disagree. In addition data were collected on nurses' sociodemographic data including sex, marital status, age, education level, years of experience, working unit, and the most frequent shift they work.

\section{Procedures}

The Ethics Committee of Faculty of Nursing, Alexandria University has approved the study protocol. They have determined that this survey does not fall under the committee's jurisdiction. The Medication Administration Error Survey was translated into Arabic language and accordingly, minor changes were made for a few unclear words. Also it was tested for content validity by five experts in the same field of the study of nursing at Alexandria Faculty of Nursing. The subscales' reliability values of these factors measured by Cronbach's coefficient Alpha, in which the internal consistency reliability ranged from 0.75 to 0.92 , while the statistical significance level was set at $\mathrm{p}<$ 0.05 .

Before embarking to data collection, an informed consent was obtained from each participant to share in the study. All participants were assured that their participation is voluntary. Also their privacy and confidentiality were maintained. A pilot study was carried out on 10 nurses who were working in other unit rather than the studied units and the necessary modifications were made. The questionnaire was hand delivered to each study participant in the morning and afternoon shifts and it was completed through self-report method. About 30 minutes were consumed to complete the questionnaire. Data collection took about three months from June 2013 till the end of august 2013.

\section{Statistical Analysis}

After data were collected it was revised, coded and fed to statistical software SPSS IBM version 20. The given graph was constructed using Microsoft excel software. All statistical analysis was done using two tailed tests and alpha error of 0.05 . Discrete items concerning nurses responses for each domain were summed together to have the domain total score. Descriptive statistics in the form of frequencies and percent were used to describe the categorical data variables while mean and standard deviation was used to describe domains scores. To test for association between sample characteristics and their scores at different domains, independent samples t-test and One Way ANOVA were used. To identify the relative importance of each domain at committing error, factor analysis was used to express factor loading which is the correlation between each domain and the overall hidden factor.

\section{Results}

Table 1 illustrated the demographic characteristics of nursing staff at emergency department in Main University Hospital. The table reveals that the female nurses were the dominant, $85.7 \%$ of nursing staff were female, and more than half of them were in the age group from 20 to less than 30 years old, as well as $65.5 \%$ of them were married. It was observed that majority of nursing staff $(79.8 \%)$ were permanent residents at Alexandria governorate, $72.6 \%$ of them had diploma secondary school degree, and $73.8 \%$ of them had less than 10 years of experience in their work unit. Moreover, it can be noticed that $54.8 \%$ of nursing staff always work variable shifts.

\begin{tabular}{|l|l|l|}
\hline Socio demographic data & No & $\%$ \\
\hline Age & 48 & 57.1 \\
\hline - $20-$ & 48 \\
\hline
\end{tabular}


Page 3 of 7

\begin{tabular}{|l|l|l|}
\hline$\cdot 30-$ & 17 & 20.2 \\
\hline$\cdot 40+$ & 19 & 22.6 \\
\hline
\end{tabular}

the factors loading values of MAEs, participants reported that the physician-nurses' communication reasons $(0.84)$ was perceived as the most important factor for reasons of MAEs occurrence. These included illegible physicians' medication orders, unclear physicians' medication, frequently change physicians' orders, poor communication between nurses and physicians. The second factor was medication packaging (0.79) such as look-alike/sound-alike medication names can result in medication errors. Misreading medication names that look similar is a common mistake. These look-alike medication names can lead to errors associated with verbal prescriptions. The third factor was pharmacy processes reason (0.76) for example, pharmacy delivers incorrect doses, and pharmacy does not prepare and label the medication correctly, as well as pharmacists unavailable 24 hours a day. The last factor was nurse staffing (0.72) was perceived as the least reason of MAEs occurrence.

\begin{tabular}{|l|l|l|}
\hline - Daily Travel & 7 & 8.3 \\
\hline - Permanent Residence & 67 & 79.8 \\
\hline - Temporary Residence & 10 & 11.9 \\
\hline Education & \multicolumn{2}{|l}{} \\
\hline - Diploma Secondary School & 61 & 72.6 \\
\hline - Nursing Institute & 12 & 14.3 \\
\hline - B SN & 11 & 13.1 \\
\hline
\end{tabular}

\section{Experience in Nursing (in years)}

\begin{tabular}{|l|l|l|}
\hline$\cdot<10$ & 38 & 45.2 \\
\hline$\cdot 10-19$ & 24 & 28.6 \\
\hline$\cdot 20+$ & 22 & 26.2 \\
\hline
\end{tabular}

Experience in The Hospital (in years)

\begin{tabular}{|l|l|l|}
\hline$\cdot<10$ & 42 & 50 \\
\hline$\cdot 10-19$ & 21 & 25 \\
\hline$\cdot 20+$ & 21 & 25 \\
\hline
\end{tabular}

\section{Experience in the Unit (in years)}

\begin{tabular}{|l|l|l|}
\hline$<10$ & 62 & 73.8 \\
\hline$\cdot 10-19$ & 13 & 15.5 \\
\hline$\cdot 20+$ & 9 & 10.7 \\
\hline
\end{tabular}

Shift

\begin{tabular}{|l|l|l|}
\hline - Morning & 33 & 39.3 \\
\hline - Evening & 2 & 2.4 \\
\hline - Night & 3 & 3.6 \\
\hline - Variable & 46 & 54.8 \\
\hline
\end{tabular}

Table 1: Distribution of nursing staff demographic characteristics at Main University Hospital Emergency Department

Figure 1 represents the perception of participants for why medication administration errors occur. Accordingly the ranking of

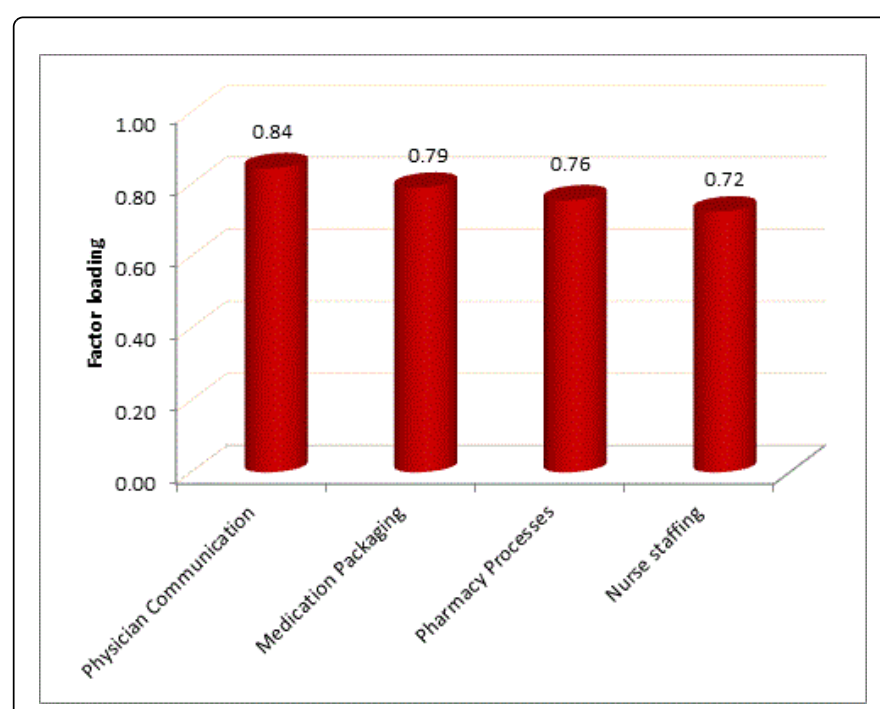

Figure 1: Relative ranks for causes of committing drug medication error according to nurses' perception

Table 2 describes the relationship of socio-demographic data with the four reasons of why medication administration errors occur. This study revealed no statistically significant relation between participants' sex, residence, and educational level and their experience in units with reasons of why MAEs occur. Concerning age, there were significant relation with only medication packaging reasons of MAEs at $\mathrm{P}<0.05$. Regarding marital status, there was statistically significant relationship between participants and physician - nurse communication reason $\mathrm{P}<0.05$ where the highest mean percent for single nurses agree that these reasons causing MAEs as well as statistically significant correlation for medication package reason of why MAEs occur $\mathrm{P}<0.05$.In relation to nurses' experience, there were inverse statistically significant correlation between nurses experience for medication packing reasons of why MAEs occur at $\mathrm{P}<0.05$. Furthermore, this study showed statistically significant correlation between participants' most frequent shift with medication packaging reasons of why MAEs occur $\mathrm{P}<0.05$. 
Citation: El Seesy N, El Sebaey F (2015) Emergency Department Nurses' Perceptions toward Factors Influencing the Occurrence of Medication Administration Errors. J Nurs Care 4: 289. doi:10.4172/2167-1168.1000289

Page 4 of 7

\begin{tabular}{|c|c|c|c|c|c|c|c|c|c|c|c|c|}
\hline \multirow{2}{*}{$\begin{array}{l}\text { Socio Demographic } \\
\text { characteristics }\end{array}$} & \multicolumn{2}{|c|}{$\begin{array}{l}\text { Physician } \\
\text { communication }\end{array}$} & \multirow{2}{*}{\begin{tabular}{|l|}
$\mathbf{P}$ \\
Mean
\end{tabular}} & \multicolumn{2}{|c|}{$\begin{array}{l}\text { Medication } \\
\text { packaging }\end{array}$} & \multirow{2}{*}{$\begin{array}{l}P \\
S D\end{array}$} & \multicolumn{2}{|c|}{$\begin{array}{l}\text { Pharmacy } \\
\text { processes }\end{array}$} & \multirow{2}{*}{$\mathbf{P}$} & \multicolumn{2}{|c|}{ Nurse staffing } & $\mathbf{P}$ \\
\hline & Mean & SD & & SD & Mean & & Mean & SD & & & & \\
\hline \multicolumn{13}{|l|}{ Age } \\
\hline 20- & 53.5 & 23.9 & \multirow{3}{*}{0.581} & 68.6 & 30 & \multirow{3}{*}{$0.006^{*}$} & 71.9 & 31.7 & \multirow{3}{*}{0.197} & 44.3 & 23.2 & \multirow{3}{*}{0.147} \\
\hline $30-$ & 52 & 21.7 & & 52.5 & 33.3 & & 69.9 & 31.7 & & 51.2 & 21.4 & \\
\hline $40+$ & 46.3 & 31.3 & & 41.7 & 33.6 & & 86 & 28.4 & & 56.6 & 27.3 & \\
\hline \multicolumn{13}{|l|}{ Marital Status } \\
\hline Single & 59.2 & 24.5 & \multirow{2}{*}{$0.043^{*}$} & 71.3 & 26.3 & \multirow{2}{*}{$0.015^{*}$} & 73.2 & 30.1 & \multirow{2}{*}{0.753} & 47 & 23.6 & \multirow{2}{*}{0.685} \\
\hline Married & 47.5 & 24.8 & & 52.9 & 34.8 & & 75.5 & 32 & & 49.2 & 24.5 & \\
\hline \multicolumn{13}{|l|}{ Sex } \\
\hline Male & 40 & 21.5 & \multirow{2}{*}{0.086} & 50 & 24.9 & \multirow{2}{*}{0.301} & 74.5 & 26.6 & \multirow{2}{*}{0.987} & 56.3 & 23.3 & \multirow{2}{*}{0.229} \\
\hline Female & 53.5 & 25.4 & & 60.8 & 34.2 & & 74.7 & 32.1 & & 47.2 & 24.1 & \\
\hline \multicolumn{13}{|l|}{ Residence } \\
\hline Daily Travel & 46.2 & 24.6 & \multirow{3}{*}{0.185} & 58.3 & 27.2 & & 81 & 16.3 & & 45.8 & 28.3 & \\
\hline Permanent Residence & 50.1 & 25.1 & & 56.5 & 33.9 & 0.151 & 73.8 & 32.7 & 0.84 & 49.6 & 24.8 & 0.658 \\
\hline Temporary Residence & 65 & 23.9 & & 78.3 & 27.6 & & 76.1 & 30.2 & & 42.5 & 15.7 & \\
\hline Education & & & & & & & & & & & & \\
\hline $\begin{array}{l}\text { Diploma } \\
\text { School }\end{array}$ & 52.2 & 26.7 & & 58.3 & 33.7 & & 75.3 & 32.7 & & 51.5 & 25 & \\
\hline Nursing Institute & 50.8 & 26.1 & 0.899 & 71.5 & 33.2 & 0.303 & 84.7 & 20.4 & 0.16 & 41.7 & 22.6 & 0.164 \\
\hline BSN & 48.5 & 14.9 & & 50.8 & 28.7 & & 60.1 & 29 & & 39 & 16.7 & \\
\hline Experience in Nursing & & & & & & & & & & & & \\
\hline$<10$ & 51.8 & 24.1 & & 65.6 & 29.7 & & 70.5 & 31.4 & & 44.7 & 23.8 & \\
\hline 19-Oct & 55.1 & 23.5 & 0.576 & 66.7 & 33.6 & $0.006^{*}$ & 71.8 & 33.5 & 0.188 & 47.7 & 22.6 & 0.236 \\
\hline $20+$ & 47.3 & 29.1 & & 40.2 & 32.2 & & 85.1 & 26.9 & & 55.7 & 25.6 & \\
\hline Experience in The Hospital & & & & & & & & & & & & \\
\hline$<10$ & 51.3 & 23.7 & & 62.7 & 30 & & 68.5 & 32.4 & & 44.3 & 23.7 & \\
\hline 19-Oct & 56.3 & 23.4 & 0.5 & 70.2 & 34.2 & $0.010^{*}$ & 75.7 & 30.6 & 0.11 & 49.4 & 21.8 & 0.205 \\
\hline $20+$ & 47.1 & 29.9 & & 41.3 & 32.5 & & 86 & 27.3 & & 55.8 & 26.2 & \\
\hline Experience in the Unit & & & & & & & & & & & & \\
\hline$<10$ & 51.7 & 25.5 & & 60.5 & 32.1 & & 71 & 33.1 & & 48.1 & 24.4 & \\
\hline 19-Oct & 55.6 & 22.4 & 0.594 & 63.5 & 35.9 & 0.356 & 77.8 & 26.1 & 0.077 & 39.7 & 18.3 & 0.065 \\
\hline $20+$ & 44.4 & 27.9 & & 44.4 & 36.1 & & 95.7 & 9.5 & & 63.9 & 24.3 & \\
\hline Shift & & & & & & & & & & & & \\
\hline Morning & 48.8 & 27.5 & & 46 & 31.7 & & 81.6 & 28.5 & & 53.9 & 26.3 & \\
\hline Evening & 41.7 & 35.4 & & 50 & 23.6 & & 58.3 & 58.9 & & 37.5 & 29.5 & \\
\hline
\end{tabular}




\begin{tabular}{|c|c|c|c|c|c|c|c|c|}
\hline Night & 75.6 & 33.7 & 83.3 & 22 & 100 & 0 & 51.4 & 12 \\
\hline Variable & 52.4 & 22.5 & 67.6 & 32.2 & 68.7 & 31.9 & 44.8 & 22.6 \\
\hline
\end{tabular}

Table 2: Distribution of the studied nurses according to their socio demographic characteristics in relation to medication administration errors causes domains

\section{Discussion}

In this study, results of the factor analysis ranked four categories of factors influencing the occurrence of MAEs in emergency department. In descending order of magnitude, these categories included physician communication, followed by medication package, then pharmacy processing and finally nurse staffing. Finding of the current study illustrated that participants perceived physician communication reason as the highest ranked factor influencing the occurrence of MAEs. This finding was relevant and consistent with Dumo in 2012 [19] who reported that poor nurse/physician relationships may cause MAEs due to physicians don't spend enough time discussing care options with nurses. Many nurses still feel that physicians don't understand, respect, or care to listen to nursing perspectives on patient care that lead to misunderstanding and conflict between nurses and physicians. While, Al-Youssif in 2013 [11] illustrated that participants perceived physician-nurse relationship reason as the fourth category of MAEs. Moreover, poor communication accounts for more than $60 \%$ of the root causes of sentinel events reported to the Joint Commission (JC) Anderson and Townsend in 2010 [20].

Participants also ranked medication package as the second factor influencing the occurrence of MAEs.This result could be in the same line with Mrayyan in 2007 [21] who suggested that the medications' labels and packages may be confusingly the healthcare personnel due to do not place important information prominently and small font size of displaying text, which may lead to poor readability. In addition, Peth in 2003 [22] in USA reported that the explosion of new drugs appearing in the marketplace has made it virtually impossible for physicians, nurses, and pharmacists to keep abreast of all of the latest data concerning the indications, contraindications, drug interactions, and adverse effects associated with each new drug. Moreover, all medications have side effects, and rare but potentially fatal side effects are unlikely to show up in preliminary clinical trials. In fact, once a medication has been removed from its packaging, it's hard to identify and can be easily confused with another one [11].

The present study factor analysis for MAEs causes ranked pharmacy processing as the third factor influencing the occurrence of MAEs in ED .Ideally, the pharmacist should collaborate with the prescriber in developing, implementing, and monitoring a therapeutic plan to produce defined therapeutic outcomes for the patient.

Surprising that nurse staffing was the least ranked factor influencing the occurrence of MAEs although inadequate staffing mentioned by Egyptian nurses due to high nurse/patient ratio in ED. This finding is consistent with Wakefield in 2000 [23], who showed that workload and type of care delivery system, and other factors such as number of consecutive hours worked, rotating shifts, staffing mix and numbers, nurse-to-patient ratios, assignment of floating nurses to unfamiliar units . In addition heavier workloads also are associated with medication errors. The nursing shortage has increased workloads by increasing the number of patients for which a nurse is responsible. Also Anderson and Townsend in 2010 [20] reported that, nurses perform many tasks that take them away from the patient's bedside, such as indirect activities, answering the telephone. Absence of nurses from the bedside is directly linked to compromised patient care. In addition, Al-Shara in 2011[24] found that the highest level of medication errors were $48.4 \%, 31.7 \%$ and $11.1 \%$ related to nurses, physicians and pharmacists, respectively. Furthermore, the leading causes of medication errors were due to heavy workload (41.4\%) and new staff (20.6\%).

Based on the nurse perception, the results of the current study showed that regarding demographic characteristics, there was no relation between participants' age, marital status and their experience in units with nurse staffing and pharmacy processing factors that influencing the occurrence of MAEs. This means that all nurses are almost ranking these factors influencing the occurrence of MAEs regardless of their age, marital status or years of experience. Also, the finding of this study showed a significant difference between nurses' marital status and physician communication as factor influencing occurrence of MAEs, since single nurses had higher ranking for this factor than married ones. This could be due to physicians either male or female underestimating nursing as profession which result in poor relationship between younger nurses and physicians however, as new graduated nurses become older and experience nurses receive more respect from them.

An interesting finding in this section of the study, in relation to nurses' experience in nursing and hospital, there were statistically significant relationship between nurses experience and medication packing reasons of why MAEs occur, in which nurses who have more working experience highly perceived that medication packaging is factor causing the occurrence of MAEs compared to nurses who have worked less years of experience. This result may note that experience is important factor affect the occurrence of MAEs. This result is consistent with the study of Flor et al in 2012, Zein Eldin and Abd Elaal in $2013[25,26]$.This result is in contrast with Al-Youssif et al. in 2013 [11] who reported that there was no relation between participants' experience with reasons of why MAEs occur or not reporting.

This study showed statistically significant correlation between participants' most frequent shift with medication packaging reasons of why MAEs occur where nurses who are working night shift has highest mean for perception that medication packaging is the most factor causing MAEs. Anderson and Townsend in 2010 [20] mentioned that fatigue and sleep deprivation are linked to decreases in vigilance, memory, information processing, reaction time, and decision making. A person who works a 12-hour shift and has a long commute may need to stay awake for up to 18 consecutive hours. Moreover, Tully in 2009 and Hartel et al in 2011 [27,28] stated that fatigue and sleep deprivation also may diminish a nurse's ability to recognize subtle patient changes. As a result, the nurse may not notice an adverse reaction to a drug quickly enough to avoid a devastating outcome. When medication errors occur, multifactorial causes in a badly shaped 
system affect their occurrence, as well as manual prescriptions, lack of bar codes, stress, fatigue, lack of attention and lack of ability.

\section{Conclusion}

In conclusion, this study determined the factors influencing the occurrence of medication administration errors, as perceived by nurses in emergency department. The data of this study suggested the ranking of four reasons of why MAEs occur; nurse - physician communication reasons, medication packaging, pharmacy reasons and finally nurse staffing. Additionally, medication errors are common in emergency department. Actually, reducing these errors requires the commitment of everyone with a stake in keeping patients safe. The physician who wrote the prescribed medications, pharmacist who dispensed it and the nurse who received the medications and administered to the patient, all play an important role in preventing MAEs.

\section{Recommendations}

The current study suggested the need to improve factors influencing MAEs by nurses through; Designing safe work environment that encourage good physician-nurses' communication conducive for patient care delivery and reduce the occurrence of MAEs, healthcare organizations should ensure that all medications are provided in clearly labelled unit-dose packages for institutional use. Top management should provide adequate staffing and fair scheduling for all urgent care to provide fair workload between nursing staff that reduce the occurrence of MAEs. Developing and disseminating the patient safety guidelines in all hospital setting especially in nursing and pharmacy departments. The researchers recommended for provision of on-going education \& training on practice of safe medication administration for all nurses especially in urgent care units.

In summary, the findings from this study highlight the need to further examination on how hospital management is addressing the problem of MAEs occurrence in ED and the role of the nurse managers in preventing medication errors through participation in quality management processes. Alternate methods may need to be created for a supportive unit culture that encourages multidisciplinary team from nurse, physician, and pharmacist to prevent medication errors and improve patient safety in the ER hospital setting.

\section{References}

1. Wakefield BJ, Wakefield DS, Uden-Holman T, Blegen MA (1998) Nurses' perceptions of why medication administration errors occur. Medsurg Nurs 7: 39-44.

2. Sanghera IS, Franklin BD, Dhillon S (2007) The attitudes and beliefs of healthcare professionals on the causes and reporting of medication errors in a UK Intensive care unit. Anaesthesia 62: 53-61.

3. Peris-Lopez P, Orfila A, Mitrokotsa A, van der Lubbe JC (2011) A comprehensive RFID solution to enhance inpatient medication safety. Int J Med Inform 80: 13-24.

4. Flynn EA, Barker K, Barker B (2010) Medication-administration errors in an emergency department. Am J Health Syst Pharm 67: 347-348.

5. Rogers AE, Hwang WT, Scott LD, Aiken LH, Dinges DF (2004) The working hours of hospital staff nurses and patient safety. Health Aff (Millwood) 23: 202-212.

6. McGillis Hall L, Doran D, Pink GH (2004) Nurse staffing models, nursing hours, and patient safety outcomes. J Nurs Adm 34: 41-45.

7. Whitman GR, Kim Y, Davidson LJ, Wolf GA, Wang SL (2002) The impact of staffing on patient outcomes across specialty units. J Nurs Adm 32: 633-639.
8. Spence Laschinger HK, Leiter MP (2006) The impact of nursing work environments on patient safety outcomes: the mediating role of burnout/ engagement. J Nurs Adm 36: 259-267.

9. Fairbanks RJ, Hays DP, Webster DF, Spillane LL (2004) Clinical pharmacy services in an emergency department. Am J Health Syst Pharm 61: 934-937.

10. Mostafaei D, Barati Marnani A2, Mosavi Esfahani H3, Estebsari F4, Shahzaidi S5, et al. (2014) Medication errors of nurses and factors in refusal to report medication errors among nurses in a teaching medical center of iran in 2012. Iran Red Crescent Med J 16: e16600.

11. Al-Youssif S, Mohamed L, Mohamed N (2013) Nurses' Experiences toward Perception of Medication Administration Errors Reporting. Journal of Nursing and Health Science 1: 56-70

12. Abou Hashish E, El-Bialy G (2013) Nurses' Perceptions of Safety Climate and Barriers to Report Medication Errors. Life Science Journal 10: 2160-2168.

13. Baker M, Attala H (2012) Medications errors, causes, and reporting behaviors as perceived by nurses. Journal of pharmaceutical and biomedical sciences 19: 1-7.

14. Lisa D (2009) Medication error reporting by physicians, nurses, and pharmacists in a Level 1 Trauma Center Emergency Department.

15. Kamel S (2008) Studying medication administration errors in Ain Shams University Hospital. MSCs. Thesis. Faculty of Medicine Ain Shams University, Egypt.

16. Abo El-Maged N, Gaber E, El-Maghraby M (2002) Relationship between work setting and the occurrence of medication errors among nurses of Assiut University Hospital, Egypt. Assuit Med.J 26: 55- 66.

17. Mousa S (2000) Assessment of nursing medication errors factors causing them in the critical care unit At El Manial University Hospital, Egypt.

18. Abdou H, Saber K (2011). A baseline assessment of patient safety culture among nurses at Student University Hospital. World Journal of Medical Sciences 6: 17-26

19. Dumo MA (2012) Factors Affecting Medication Errors among Staff Nurses: Basis in the Formulation of Medication Information Guide. IAMURE International $\mathrm{J}$ of Health Education 1: 1-62.

20. Anderson P, Townsend T (2010) Medication errors: Don't let them happen to you Mistakes can occur in any setting, at any step of the drug administration continuum. Here's how to prevent them. American Nurse Today 5: 23-28.

21. Mrayyan MT, Shishani K, Al-Faouri I (2007) Rate, causes and reporting of medication errors in Jordan: nurses' perspectives. J Nurs Manag 15: 659-670.

22. Peth HA (2003) Medication errors in the emergency department: a systems approach to minimizing risk. Emerg Med Clin North Am 21: 141-158.

23. Wakefield J B, Uden-Holman T, and Wakefield S D (2000) Development and Validation of the Medication Administration Error Reporting Survey, Journal of Advances in Patient Safety 4: 475-89.

24. Al-Shara M (2011) Factors contributing to medication errors in Jordan: a nursing perspective. Iran J Nurs Midwifery Res 16: 158-161.

25. N. Flor (2012) Ateneo de Manila University. Retrieved February 2012, from Ateneo de Manila University

26. Zein ElDin YK, Abd ElAal HN (2013) The Relationship between Perceived Safety Climate, Nurses' Work Environment and Barriers to Medication Administration Errors Reporting. Life Science Journal, 10: 950-62.

27. Tully MP, Ashcroft DM, Dornan T, Lewis PJ, Taylor D, et al. (2009) The causes of and factors associated with prescribing errors in hospital inpatients: a systematic review. Drug Saf 32: 819-836.

28. Hartel MJ, Staub LP, Röder C, Eggli S (2011) High incidence of medication documentation errors in a Swiss university hospital due to the handwritten prescription process. BMC Health Serv Res 11: 199. 